\title{
Stimulation of RTH1 Nuclease of the Yeast Saccharomyces cerevisiae by Replication Protein A
}

\author{
Esther E. Biswas, Fan Xiu Zhu, and Subhasis B. Biswas* \\ Department of Molecular Biology, Science Center Room 305A, University of Medicine \& Dentistry of New Jersey, \\ Stratford, New Jersey 08084
}

Received November 22, 1996; Revised Manuscript Received March 19, $1997^{\circledR}$

\begin{abstract}
The RTH1 nuclease is involved in the replication of chromosomal DNA as well as in the repair of DNA damage. Replication protein A (RPA) is also an integral part of the DNA replication and repair processes. We have investigated the roles(s) of RPA in the function of RTH1 nuclease, including its structure specific endonuclease activity. Initial in vitro studies, which employed a "flap" or a "pseudo Y" substrate containing a short $14 \mathrm{bp}$ duplex region, showed the effect of RPA to be minimal or inhibitory. As RPA inhibition is unwarranted for a protein participating in the DNA replication process, we have further investigated the mechanism of such inhibition. Alternate flap and pseudo Y substrates with a long duplex region (50 bp) were prepared using M13mp19 ssDNA and synthetic oligonucleotides. Yeast RPA stimulated the endonuclease activity of $R T H 1$ endonuclease with these substrates in a dose-dependent manner. Kinetic analysis suggested that yRPA exerted a bipartite effect on the nuclease reaction: (i) the "load time" of RTHI nuclease onto the DNA substrate decreased from $\sim 5$ to $2 \mathrm{~min}$ in the presence of RPA, and (ii) following initiation of the nuclease reaction, the initial rate of the reaction increased 10fold in the presence of yRPA. Further analysis of the interaction of RPA with various endonuclease substrates indicated that RPA has a weak helix destabilizing effect and could melt small, $14 \mathrm{bp}$, regions of duplex DNA. RTHI endonuclease cleaves the DNA strand at the junction of single- and doublestranded DNA; consequently, the observed inhibition with small duplex substrates was likely due to duplex melting. Our studies also demonstrated that RPA stimulated the RNase H activity of RTH1 nuclease significantly. In both instances ( $R T H I$ endonuclease and $\mathrm{RNase} \mathrm{H})$, the stimulation may involve a specific interaction of RPA with the RTHI nuclease rather than a structural positioning of the DNA substrate by RPA.
\end{abstract}

Chromosomal DNA replication or repair of damaged DNA requires removal of the DNA lesion by nucleases; frequently, these are $5^{\prime} \rightarrow 3^{\prime}$ exonucleases and endonucleases (Prakash et al., 1993; Kornberg \& Baker, 1992). A family of eukaryotic nucleases with endo- and $5^{\prime} \rightarrow 3^{\prime}$ exonucleases activities have been identified in lower as well as higher eukaryotes (Goulian et al., 1990; Ishimi et al., 1988; Waga et al., 1994; Turchi et al., 1994; Harrington \& Lieber, 1994; Murray et al., 1994; Sommers et al., 1995). The first evidence of involvement of a $5^{\prime} \rightarrow 3^{\prime}$ exonuclease and its involvement with DNA polymerase $\alpha$-primase (pol $\alpha$ ) in DNA replication was shown by Goulian et al. (1990) in a pol $\alpha$-primase-dependent replication using a single-stranded circular DNA as a template. The RAD2 gene product of the yeast Saccharomyces cerevisiae has been shown by Prakash and co-workers to be involved in the repair of UV damaged DNA and is one of the first known eukaryotic nucleases of this class (Habraken et al., 1993, 1994, 1995; Guzder et al., 1995). The human homolog of xeroderma pigmentosum group $\mathrm{G}$ protein (XPG) protein is also a $5^{\prime} \rightarrow$

\footnotetext{
* Corresponding author.

${ }^{\otimes}$ Abstract published in Advance ACS Abstracts, May 1, 1997.

${ }^{1}$ ABBREVIATIONS: ATP, adenosine triphosphate; ATPase, adenosine triphosphatase; BSA, bovine serum albumin; DTT, dithiothreitol; EDTA, ethylenediaminetetraacetic acid; PMSF, phenylmethanesulfonyl flouride; pol $\alpha$, DNA polymerase $\alpha$; RPA, replication protein A; ssDNA, single-stranded DNA; dsDNA, double-stranded DNA; TLCK, $p$-tosyllysyl chloromethyl ketone; TPCK, $p$-tosylphenylalanine chloromethyl ketone; Tris, tris(hydroxymethyl)aminomethane.
}

$3^{\prime}$ exonuclease. These nucleases have been implicated in a number of other putative DNA repair and replication functions. Similar nucleases have been described previously in prokaryotic organisms such as the $5^{\prime} \rightarrow 3^{\prime}$ exonuclease of DNA polymerase I of Escherichia coli (Kornberg \& Baker, 1992), gene6 exonuclease of T7 bacteriophage (Gutman \& Minton, 1993), and RNase H of T4 bacteriophage (Hollingsworth \& Nossal, 1991; Mueser et al., 1996), etc. Although the eukaryotic homologs of these nucleases are generally smaller proteins, they have retained a high degree of sequence and structural homology, especially in the nuclease domain (Mueser et al., 1996; Shen et al., 1996). The S. cerevisiae open reading frame (ORF) YKL510, which is now known as RTH1 or RAD2 homolog, is highly homologous in structure and function to the murine flap endonuclease (FEN-1) and human FEN-1 (also known as maturation factor 1 or MF-1) (Harrington \& Lieber, 1994; Murray et al., 1994, Waga et al., 1994).

The presence of nucleases that interact with DNA polymerases has been demonstrated by various laboratories (Goulian et al., 1990; Waga et al., 1994). Earlier we have shown that a $5^{\prime} \rightarrow 3^{\prime}$ exonuclease copurifies with DNA polymerase $\alpha$-primase (pol $\alpha$ ) complex (Biswas et al., 1993a). Purification of this nuclease from $S$. cerevisiae and determination of its identity as the RTH1 $5^{\prime} \rightarrow 3^{\prime}$ exonuclease have been described earlier (Zhu et al., 1997). The native $R T H 15^{\prime} \rightarrow 3^{\prime}$ exonuclease also possesses a structure-specific 
endonuclease activity on forked DNA structures, in addition to its exonuclease and RNase $\mathrm{H}$ activities.

Replication protein A has been shown to be an important component of the DNA replication apparatus, and it stimulates and interacts with various components of the replication apparatus (Wold et al., 1987; Wold \& Kelly, 1988; Heyer et al., 1989, 1990; Erdile et al., 1991) as well as DNA repair and recombination (Alani et al., 1992). A major role of the $R T H 1$ nuclease is in the DNA replication process, and thus, an important question regarding the mechanism of action of the exonuclease is the role of replication protein A (RPA), if any, in the modulation of the RTHI nuclease activities. Thus far, there is no clear understanding of the role of RPA. We have investigated the role of RPA in the RTHI nuclease function using purified yeast and human (HeLa) RPA and purified $R T H I$ nuclease from yeast as described (Zhu et al., 1997).

\section{MATERIALS AND METHODS}

Yeast. Protease deficient yeast, S. cerevisiae, BJ 2168 strain from the Yeast Genetic Stock Center (Berkeley, CA) was grown in a laboratory fermenter to midlog phase, and the cells were chilled to $4{ }^{\circ} \mathrm{C}$ then harvested by centrifugation. The cells were then resuspended in $50 \mathrm{mM}$ Tris- $\mathrm{HCl}$ (pH 7.5) containing $1 \mathrm{mM}$ EDTA and $10 \%$ glycerol to OD $400(\lambda=600 \mathrm{~nm})$ and stored frozen at $-80^{\circ} \mathrm{C}$ until further use.

Nucleic Acids, Enzymes, and Other Reagents. Ultrapure deoxy- and ribonucleotides were obtained from Pharmacia (Piscataway, NJ) and were used without further purification. $\left[\alpha-{ }^{32} \mathrm{P}\right] \mathrm{ATP},\left[\alpha-{ }^{32} \mathrm{P}\right] \mathrm{dATP}$, and $\left[\gamma_{-}{ }^{32} \mathrm{P}\right] \mathrm{PTP}$ were obtained from DuPont/NEN (Boston, MA). yRPA used in this study was purified as described (Biswas et al., 1993a). hRPA was purified from $\mathrm{HeLa}$ cells following a procedure similar to that described by Wold and Kelly (1988). The native yeast RTHI nuclease utilized in these studies was purified as described in the preceding paper. Terminal deoxynucleotidyl transferase was purchased from United States Biochemical Corp. (Cleveland, OH). T4 polynucleotide kinase was obtained from New England Biolabs (Beverly, MA). All chemicals used to prepare buffers and solutions were reagent grade and were purchased from Fisher Chemical Company (Pittsburgh, PA). Protease inhibitors were from Bachem (Los Angeles, CA).

Oligonucleotides. Oligonucleotides were synthesized at commercial facilities either from Oligos Inc. (Portland, OR) or Integrated DNA Technologies (Coralville, IA). The oligonucleotides were analyzed and further purified by polyacrylamide $7 \mathrm{M}$ urea gel electrophoresis. The final purity of the oligonucleotides were $>99 \%$ as judged by autoradiography of the phosphorylated products. The sequences of the oligonucleotides used in this study were as follows: (1) 5'-GGGTCTCACGACGTTGTAAAACGACGGCCAGTGAATTCGAGCTCGGTACCCGGGGTAGGA3'; (2) 5'-TTAAGTTGGGTAACGCCAGGGTTTTCCCAG3'; (3) 5'-GGACTCTGCCTCAAGACGGTAGTCAACGTG$3^{\prime} ;$ (4) 5'-GATGTCAAGCAGTCCTAACTTTGAGGCAGAGTCC-3'; (5) 5'-CACGTTGACTACCGTC-3'.

Oligonucleotides $\mathbf{1}$ and $\mathbf{2}$ were used to prepare substrate for exonuclease and endonuclease activities by annealing to M13mp19 ssDNA to construct M13 flap (substrate A) and M13 pseudo Y (substrate B). Oligonucleotides $3-5$ were used to prepare flap (substrate C) and pseudo Y (substrate
A.
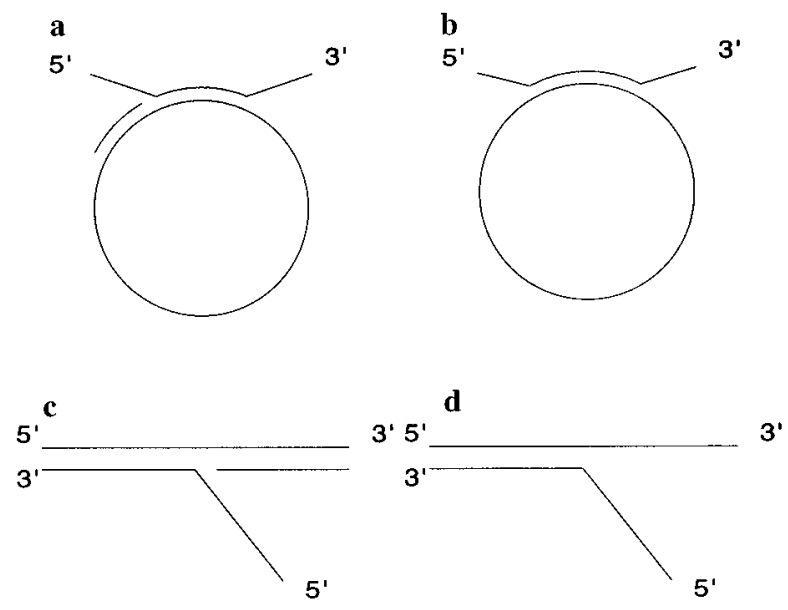

B.
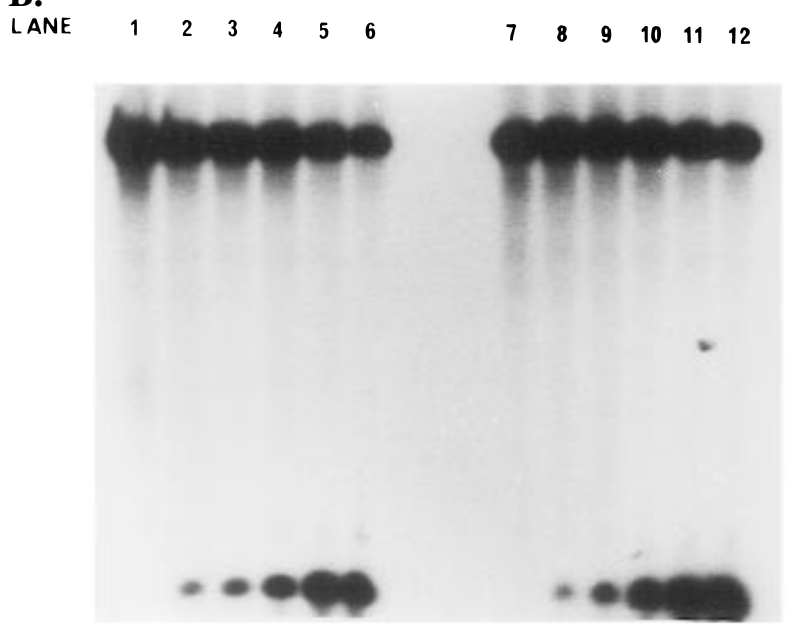

FIGURE 1: (A) Schematic representation of endo- and exonuclease substrates utilized in this study. Details on the preparation of these substrates are given in Materials and Methods. (a) M13 flap substrate, (b) M13-Y substrate, (c) flap substrate and (d) pseudo-Y substrate. (B) Activity of RTH1 nuclease on M13 flap (substrate a) and M13-Y (substrtae b). A standard endonuclease assay was carried out with 100 pmol of either M13-Y (lanes 1-6) or M13 flap (lanes 7-12) substrates in the presence of increasing amounts of RTH1. Lane 1, blank, no RTH1; lane 2, $24 \mathrm{pg}$; lane 3, $48 \mathrm{pg}$; lane 4, $120 \mathrm{pg}$; lane 5, $240 \mathrm{pg}$; lane 6, $480 \mathrm{pg}$; lane 7, blank (no RTH1); lane 8, $24 \mathrm{pg}$; lane 9, $48 \mathrm{pg}$; lane 10, $120 \mathrm{pg}$; lane 11, 240 pg; lane 12, $480 \mathrm{pg}$.

D) substrates as described by Harrington and Lieber (1994). A schematic diagram of these substrates are shown in Figure 1 .

Buffers. Buffer E contained $25 \mathrm{mM}$ Tris- $\mathrm{HCl}(\mathrm{pH} 7.5)$, $10 \%(\mathrm{v} / \mathrm{v})$ glycerol, $0.1 \mathrm{mg} / \mathrm{mL}$ BSA, and $5 \mathrm{mM}$ DTT. Buffer $\mathrm{F}$ contained $95 \%$ formamide, $10 \mathrm{mM}$ EDTA, $1 \mathrm{mg} /$ $\mathrm{mL}$ bromophenol blue, and $1 \mathrm{mg} / \mathrm{mL}$ xylene cyanol.

Preparation of Endonuclease Substrates. (i) M13 Flap Substrate. Oligonucleotide $\mathbf{1}$, which is a 60 -mer complementary to a $50 \mathrm{bp}$ sequence between nucleotides 6268 and 6317 of M13mp19 ssDNA was labeled at its 5' end using T4 polynucleotide kinase. The oligonucleotide was hybridized to M13mp19 as previously described (Biswas et al., 1993b). Excess unhybridized labeled oligonucleotide was removed by spin column (Promega, Madison, WI) purification. The purified substrate was diluted to $17 \mathrm{fmol} / \mu \mathrm{L}$ $(10000-20000 \mathrm{cpm} / \mu \mathrm{L})$ with $10 \mathrm{mM}$ Tris- $\mathrm{HCl}(\mathrm{pH} 7.5)$ and $1 \mathrm{mM}$ EDTA. 
(ii) M13 Y Substrate. This substrate was prepared exactly as described for the forked substrate except that a $50 \mathrm{bp}$ oligonucleotide, oligo $\mathbf{2}$, with sequence complementary to the sequence between nucleotides 6268 and 6317 of M13mp19 was used.

(iii) Flap and Pseudo- Y Substrate for Endonuclease Activity. These substrates are identical to those described by Harrington and Lieber (1994). Oligonucleotides 3-5 were used to prepare these substrates following the method given by Harrington and Lieber (1994).

Endonuclease Assay. The reactions were set up on ice, and the standard reaction $10 \mu \mathrm{L}$ mixture in buffer E contained $9 \mathrm{mM} \mathrm{MgCl} 2,100 \mathrm{pmol}$ of substrate DNA, and native $R T H 1$ nuclease (Fr VI), as indicated. The reactions were incubated for $5 \mathrm{~min}$ at $37^{\circ} \mathrm{C}$ and were terminated by the addition of and equal volume of buffer $\mathrm{F}$, boiling for $3 \mathrm{~min}$, followed immediately by chilling in an ice-water bath. One-half of the reaction product was analyzed by electrophoresis on a $20 \%$ acrylamide (A:B 19:1), 7 M urea gel. Following electrophoresis, the gel was dried and exposed to Fuji RX film for $12 \mathrm{~h}$ at $-80^{\circ} \mathrm{C}$. Quantitation of the data was carried out by scanning densitometry of the autoradiogram.

Other Methods. Protein concentrations were estimated according to the method of Bradford (1976), using bovine serum albumin as a standard. The ATPase assays were carried out as previously described (Biswas et al., 1993b). Densitometry was carried out using a Molecular Dynamics scanning densitometer and Image Quant software (Sunnyvale, CA). Protein active site modeling was done using the SYBYL program developed by Tripos Associates (St. Louis, MO). We used Kollman-all-atom force field method for energy minimization.

\section{RESULTS}

Native RTH1 Endonuclease Prefers DNA Substrates Having a Flap Structural Motif. We have purified to homogeneity a $47 \mathrm{kDa}$ endo/exonuclease from the yeast $S$. cerevisiae that we previously found to be associated with a polymerase $\alpha$ multiprotein complex (Biswas et al., 1993b; Zhu et al., 1997). Peptide sequence analysis of this polypeptide indicated that its sequence is identical to that of the YKL150 gene product, which is now known as RTH1 (Sommers et al., 1995). Previous studies (Harrington \& Lieber, 1994) have clearly demonstrated DNA structure specificity of the mammalian FEN1 and recombinant yeast RTH1 endonuclease expressed in E. coli. The structures of the various $R T H 1$ nuclease substrates with and without the $5^{\prime}$ flap structural feature are shown in Figure 1. The M13 flap and M13-pseudo-Y are shown in Figure 1 as structure $\mathrm{a}$ and $\mathrm{b}$, respectively. These structures are similar to the flap and pseudo Y substrates, shown as structures $\mathrm{c}$ and $\mathrm{d}$, described earlier by Harrington and Lieber (1994), except that the length of the duplex region has been increased from 14 to $50 \mathrm{bp}$.

The activity of the RTHI nuclease on a standard endonuclease assay was carried out, with increasing amounts of RTH1 nuclease over a range 12-500 pg (Figure 1B). Endonuclease products were easily detectable with the M13 flap substrate using $24 \mathrm{pg} R T H 1$; however, higher levels of the nuclease were required for the same amount of cleavage using the pseudo $\mathrm{Y}$ substrate. The activity of RTH1 endonuclease on flap and pseudo $\mathrm{Y}$ substrates $\mathrm{c}$ and $\mathrm{d}$ were identical to that observed with M13 flap and M13 Y
LANE
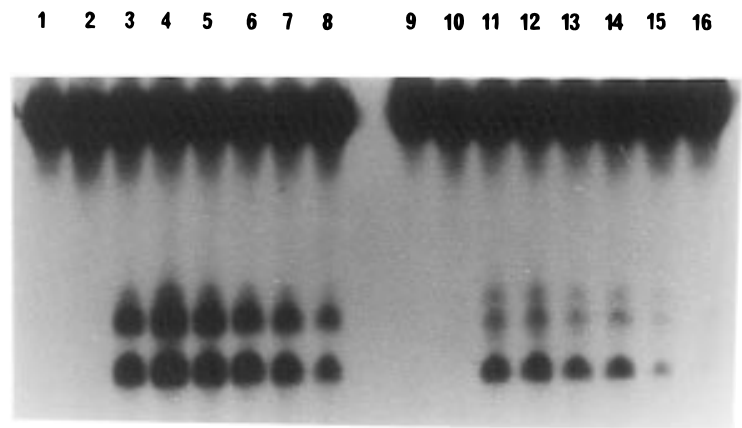

FIGURE 2: Influence of yeast RPA on the endonuclease digestion of flap (substrate c) and pseudo Y (substrate d) by native RTH1 nuclease. A standard endonuclease assay, as described in Materials and Methods, was carried out with $12 \mathrm{pg}$ of RTH1, using either the flap (lanes 1-8) or pseudo-Y (lanes 9-16) substrates in the presence of the indicated amounts of yRPA. Lanes 1 and 9, blank, no $R T H 1$; lanes 2 and 10, yRPA, $200 \mathrm{ng}$, no RTH1; lanes 3 and 11, RTH1, no yRPA; lanes 4 and 12, 10 ng of yRPA; lanes 5 and 13, $25 \mathrm{ng}$ of yRPA; lanes 6 and 14, $50 \mathrm{ng}$ of yRPA; lanes 7 and 15, $100 \mathrm{ng}$ of yRPA; lanes 8 and 16, $200 \mathrm{ng}$ of yRPA.

substrates (data not shown). Consequently, our results indicated that the preferred substrate for the native RTHI was a flap substrate, and its activity on a pseudo Y structure was less efficient. Thus, these results with native RTH1 nuclease correlated well with that of Harrington and Lieber (1994) for the recombinant enzyme expressed in E. coli.

Yeast Replication Protein A Modulated the Endonuclease Activity of RTH1. Yeast RPA has been shown to be involved in DNA replication and recombination, and stimulates various enzymes, including DNA polymerases (Wold \& Kelly, 1988; Heyer \& Kolodner, 1989; Heyer et al., 1990; Erdile et al., 1989). As RTH1 nuclease is also involved in eukaryotic DNA replication (Goulian et al., 1990; Waga et al., 1994; Sommers et al., 1995), particularly in the lagging strand DNA synthesis; we have examined the effect of RPA in modulating the activity of $R T H 1$ nuclease.

(A) RPA Effect on the RTH1 Endonuclease Activity on Substrates $c$ and $d$ Containing A Short Duplex Region. The effects of RPA on the activity of RTH1 nuclease were examined initially using flap substrate $\mathrm{c}$ and pseudo $\mathrm{Y}$ substrate $\mathrm{d}$ as these are the most commonly used substrates for RTHI endonuclease activity (Harrington \& Lieber, 1994). The results of this experiment are presented in Figure 2. A small increase in the RTHI nuclease activity was seen at a very low concentration of RPA; however, titration of RPA revealed that it could strongly inhibit the endonuclease activity of RTH1 nuclease on either of these two substrates. Inhibition to such an extent was unanticipated, and thus, we further investigated the mechanism of RPA inhibition of endonuclease activity.

Single-stranded DNA binding proteins (SSBs) do not usually have any helix destabilizing activity, particularly with long duplex DNA templates. In our previous studies involving DNA helicases, we have observed a very weak helix destabilizing activity with yeast RPA (E. Biswas, unpublished observation). However, the activity is weak enough so that it does not destabilize DNA substrates with longer duplex regions. It was perhaps then possible that RPA inhibited the endonuclease activity by destabilizing the small duplex (14 bp) regions in substrates $\mathrm{c}$ and $\mathrm{d}$ (Figure 1A). Consequently, we have analyzed the effect of RPA on both of these substrates. The substrates were incubated with increasing amounts of yeast RPA at $30^{\circ} \mathrm{C}$, and the reaction 


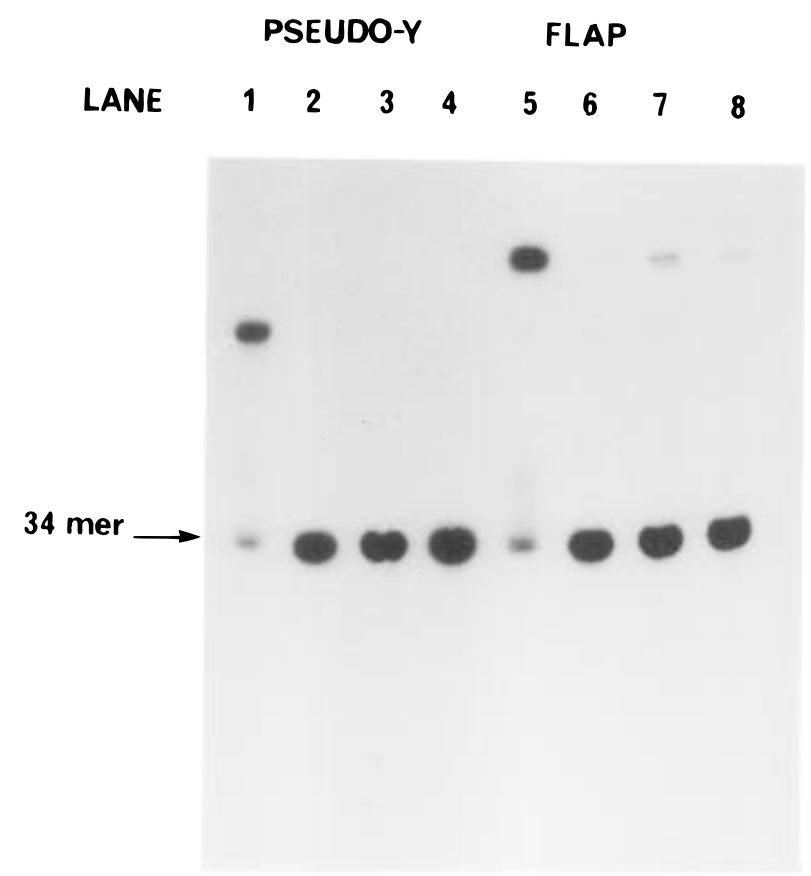

FIGURE 3: Stability of pseudo Y and flap substrates in the presence of yRPA. Standard reaction mixtures were set up containing either the flap or pseudo Y substrate, in the absence of any RTH1 protein. The reactions were then incubated at $37{ }^{\circ} \mathrm{C}$ for 5 min after which they were analyzed on a $20 \%$ acrylamide, $0.1 \%$ SDS, $1 \times$ TBE gel. The autoradiogram of the dried gel is shown above. Lane 1, pseudo Y substrate; lane 2, heat denatured pseudo Y substrate; lane 3, pseudo y substrate incubated in the presence of $50 \mathrm{ng}$ of yRPA; lane 4, pseudo Y substrate incubated in the presence of 200 ng yRPA. lane 5, flap substrate; Lane 6, heat denatured flap substrate; lane 7, flap substrate incubated in the presence of $50 \mathrm{ng}$ of yRPA; lane 8, flap substrate incubated in the presence of 200 ng of yRPA.

products were analyzed on a SDS-PAGE gel (20\% polyacrylamide gel; with a running buffer containing $89 \mathrm{mM}$ Trisborate, $\mathrm{pH} 8.3,1 \mathrm{mM}$ EDTA, and $0.1 \%$ SDS). The results of this analysis are presented in Figure 3. These results demonstrated that the $14 \mathrm{bp}$ duplex present in either substrate $\mathrm{c}$ or substrate $\mathrm{d}$ can be easily melted by RPA alone. The endonuclease activity of $R T H 1$ has a strict structural requirement in its substrates as described by Harrington and Lieber (1994). Partial duplex structures in the substrates allow RTH1 nuclease to recognize these substrates, and as a result, melting of the partial duplex should inhibit the endonuclease activity as observed in Figure 2. Therefore, we inferred from these studies that substrates $\mathrm{c}$ and $\mathrm{d}$ are not suitable for analyzing the modulation of RTHI nuclease by RPA. Substrates such as a and $\mathrm{b}$ with much longer duplex DNA would be more suitable for this analysis.

(B) RPA Effect on the RTH1 Endonuclease Activity on M13 Substrates $a$ and $b$ with a Long Duplex Region. M13 substrates $\mathrm{a}$ and $\mathrm{b}$ have $50 \mathrm{bp}$ duplex regions, and our previous studies indicate that RPA does not melt these substrates. Figure 4 shows the results of the titration obtained when RPA was added, in amounts ranging $0-200 \mathrm{ng}$, to reactions containing either the M13 flap or the M13-Y substrate. The results presented here clearly demonstrated that RPA stimulated the endonuclease activity of $\mathrm{RTHI}$ nuclease on M13 flap a and M13 pseudo Y b substrates. The maximal RPA stimulation of the endonuclease activity on M13 flap (substrate A) was approximately 3-fold as determined by scanning densitometry of the corresponding

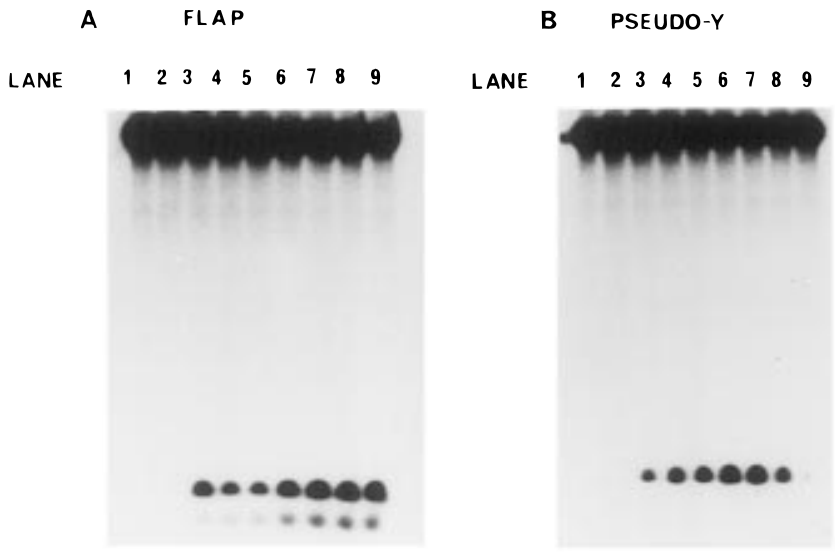

FIGURE 4: Effect of yeast RPA (yRPA) on the endonuclease activity of native $R T H 1$ Nuclease. Standard reactions were carried out which contained 100 pmol of (A) M13 flap (substrate A) and M13-Y (substrate B) with $10 \mathrm{pg}$ of native RTHI and yRPA as indicated. Incubation was at $37^{\circ} \mathrm{C}$ for $5 \mathrm{~min}$. (A) Autoradiogram corresponding to yRPA titration of reactions containing the M13 flap (substrate a). lane 1, blank , no RTH1, no yRPA; lane 2, yRPA, $200 \mathrm{ng}$, no $R T H 1$; lane 3, RTH1, no yRPA; lane 4, 10 ng of yRPA; lane 5, 25 ng of yRPA; lane 6, $50 \mathrm{ng}$ of yRPA; lane 7, $75 \mathrm{ng}$ of yRPA; lane 8, $100 \mathrm{ng}$ of yRPA; lane 9, $200 \mathrm{ng}$ of yRPA. (B) Autoradiogram corresponding to yRPA titration of reactions containing the M13-Y (substrate b). Lane 1, blank, no RTH1, no yRPA; lane 2, yRPA, 200 ng, no RTH1; lane 3, RTH1, no yRPA; lane 4, 10 ng of yRPA; lane 5, $25 \mathrm{ng}$ of yRPA; lane 6, $50 \mathrm{ng}$ of yRPA; lane 7, 75 $\mathrm{ng}$ of yRPA; lane 8, $100 \mathrm{ng}$ of yRPA; lane 9, $200 \mathrm{ng}$ of yRPA.

autoradiogram (Figure 4A). This level of stimulation was observed at about $75 \mathrm{ng}$. If the yRPA present in the reaction exceeded $100 \mathrm{ng}$, its effect was inhibitory. Similar results were obtained with the M13 Y substrate (Figure 4B). At high concentrations, RPA may be accumulating on the ssDNA portion of the substrate adjacent to the fork, thereby interfering with the recognition of the substrate by RTHI nuclease.

Enhancement of Initial Rate Kinetics by yRPA. In order to further investigate the exact nature of stimulation of $R T H I$ nuclease by yRPA, a time-course analysis of the endonuclease reaction was carried out in the presence and absence of yRPA. As shown in Figure 5, panels A and B, yRPA had a significant influence on the initial rate of the reaction. Further analysis of the data indicated that yRPA exerted a 2 -fold effect on the nuclease reaction: (i) the "load time" of $R T H 1$ onto the nuclease substrate decreased from 5 to 2 min in the presence of RPA, and (ii) following initiation of the nuclease reaction, the initial rate of the reaction was 10fold higher in the presence of yRPA.

Comparison of Stimulation of RTH1 Nuclease by Yeast and Human RPA. We have further analyzed the specificity of RPA in stimulating the endonuclease activity of $\mathrm{RTHI}$ nuclease. Human and yeast RPA were titrated in varying amounts in a standard endonuclease assay, and the results are shown in Figure 6. As previously observed, the extent of overall stimulation with yRPA was approximately 5fold. Human RPA was found to have little effect on the reaction. Figure 6 shows the results obtained when hRPA in amounts 25-200 ng were tested; however, similar results were obtained when 10 -fold higher or lower were used. Further studies are required to analyze the specificity of various ss-DNA binding proteins from heterologous sources. 

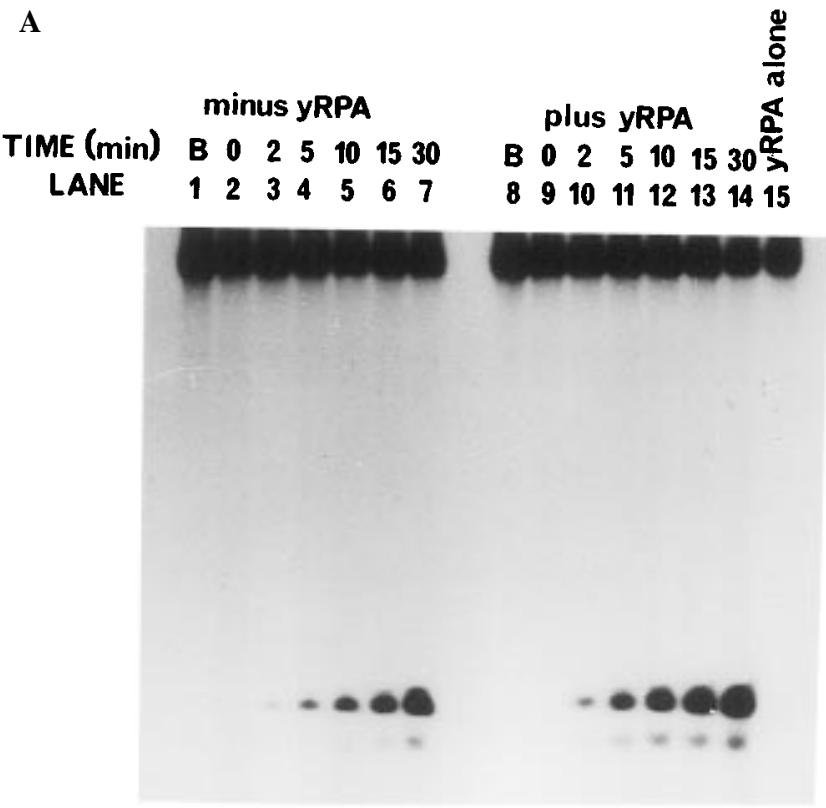

B

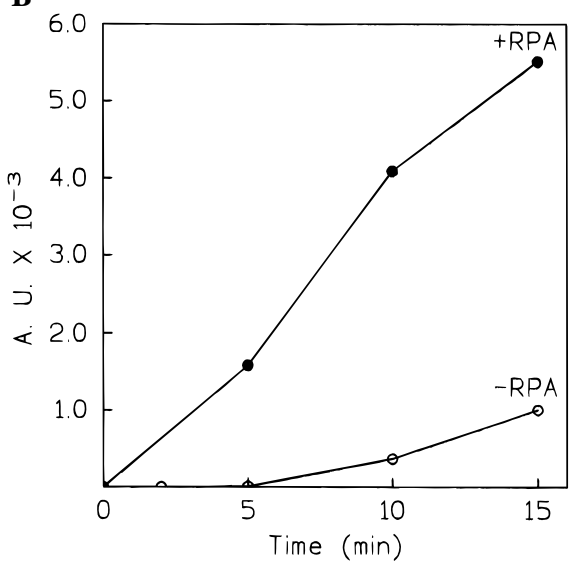

FIGURE 5: Kinetic analysis of RTH1 nuclease in the presence and absence of yeast RPA (yRPA): Standard reaction mixtures were set up containing $100 \mathrm{pmol}$ of M13 flap substrate, $12 \mathrm{pg}$ of native RTH1 and if indicated $100 \mathrm{ng}$ of yRPA. At the given time points, samples of the reaction mixture were withdrawn and the reactions terminated. (A) Autoradiogram corresponding to the time course in the presence and absence of yRPA. Lanes 1-7 reactions in the absence of yRPA. Lane 1, blank, no RTH1; lane 2, 0 min; lane 3, $2 \mathrm{~min}$; lane 4, $5 \mathrm{~min}$; lane 5, $10 \mathrm{~min}$; lane 6, $15 \mathrm{~min}$; lane 7, 30 min. lanes 8-15, reactions in the presence of $100 \mathrm{ng}$ of yRPA: lane 8, blank, no RTH1, no yRPA; lane 9, 0 min; lane 10, 2 min; lane 11, $5 \mathrm{~min}$; lane 12, $10 \mathrm{~min}$; lane 13, $15 \mathrm{~min}$; lane 14, $30 \mathrm{~min}$; lane 15, yRPA alone, no RTH1, 30 min. (B) Graphical representation of quantitation of data obtained by scanning densitometry of autoradiogram in panel $\mathrm{A}$.

Effect of RPA on the RNase H activity of RTH1 Nuclease. RTHI nuclease possesses a RNase $\mathrm{H}$ activity that is most likely important in the maturation of Okazaki fragments in the lagging strand of the replication fork, similar to that observed with the $5^{\prime} \rightarrow 3^{\prime}$ exonuclease activity of DNA polymerase I of E. coli (Kornberg \& Baker, 1992). Earlier we have shown that $R T H 1$ nuclease can degrade multimeric RNA primers synthesized by yeast pol $\alpha$-primase complex (Zhu et al., 1997). Therefore, we have examined the effect of RPA on the RNase $\mathrm{H}$ activity of RTHI nuclease. The RNA-primed DNA was prepared using pol $\alpha$-primase. RTHI nuclease digestion of the RNA-primed DNA was titrated with $0-100 \mathrm{ng}$ of RPA. The autoradiogram of the $\begin{array}{lllllllllll}\text { LANE } & 1 & 2 & 3 & 4 & 5 & 6 & 7 & 8 & 9 & 10\end{array}$

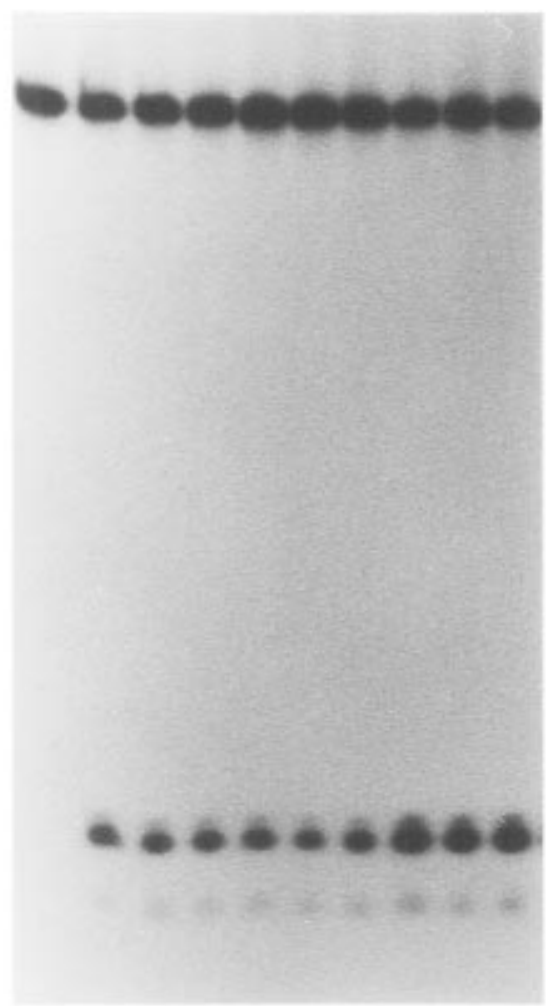

FIGURE 6: Comparison of the effects of human and yeast RPA on endonuclease activity of $R T H 1$ nuclease. A standard endonuclease assay was carried out using $12 \mathrm{pg}$ of native RTH1 using $100 \mathrm{pmol}$ of M13 flap substrate in the presence of the indicated single-stranded DNA binding proteins. lane 1, blank, no RTH1, no RPA; lane 2, $R T H 1$ alone, no RPA. Lanes 3-6, reaction in the presence of human RPA (hRPA): lane 3, $25 \mathrm{ng}$ of hRPA; lane 4, $50 \mathrm{ng}$ of hRPA; lane 5, $100 \mathrm{ng}$ of hRPA; lane 6, $200 \mathrm{ng}$ of hRPA. Lanes 7-10, reaction in the presence of yeast RPA (yRPA): lane 7, $25 \mathrm{ng}$ of yRPA; lane 8, 50 of ng yRPA; lane 9, 100 ng of yRPA; lane 10, $200 \mathrm{ng}$ of yRPA.

reaction products are shown in Figure 7. The results indicated that RPA stimulated the RNase $\mathrm{H}$ reaction of $\mathrm{RTHI}$ nuclease in a dose-dependent manner.

Putative Model of the Structure of the Active Site of RTH1 Nuclease. The primary structure of the RTHI nuclease has significant sequence homology with a number of other nucleases that are involved in DNA replication and repair including the $5^{\prime} \rightarrow 3^{\prime}$ exonuclease domain of $E$. coli DNA polymerase I, HIV reverse transcriptase, T4 RNase H and the RAD 2 family of nucleases, and xeroderma pigmentosum group G (XPG) protein (Harrington \& Lieber, 1994; Meuser et al., 1996). A sequence comparison carried out by Meuser et al. (1996) identified several residues of RTH1 nuclease that are likely involved in the nuclease function, and the most important residues are: Asp34, Asp87, Arg104, Glu156, Glu158, Asp177, and Asp179. A comparison of the structure with the active X-ray crystal structures of T4 RNase $\mathrm{H}$ (Meuser et al., 1996) and the RNase $\mathrm{H}$ domain of HIV reverse transcriptase (Kohlstaedt et al., 1992) indicated that these residues are likely involved in the coordination of two metal ions $\left(\mathrm{Mg}^{2+}\right.$ or $\left.\mathrm{Mn}^{2+}\right)$ and active site "cleft" region of the nuclease domain. On the basis of the X-ray structures of the HIV reverse transcriptase and T4 RNase H, we have used computer modeling techniques (SYBYL) to develop a putative working model of the active site of $R T H 1$ nuclease. The active site structure of the T4 RNAse $\mathrm{H}$ as determined 


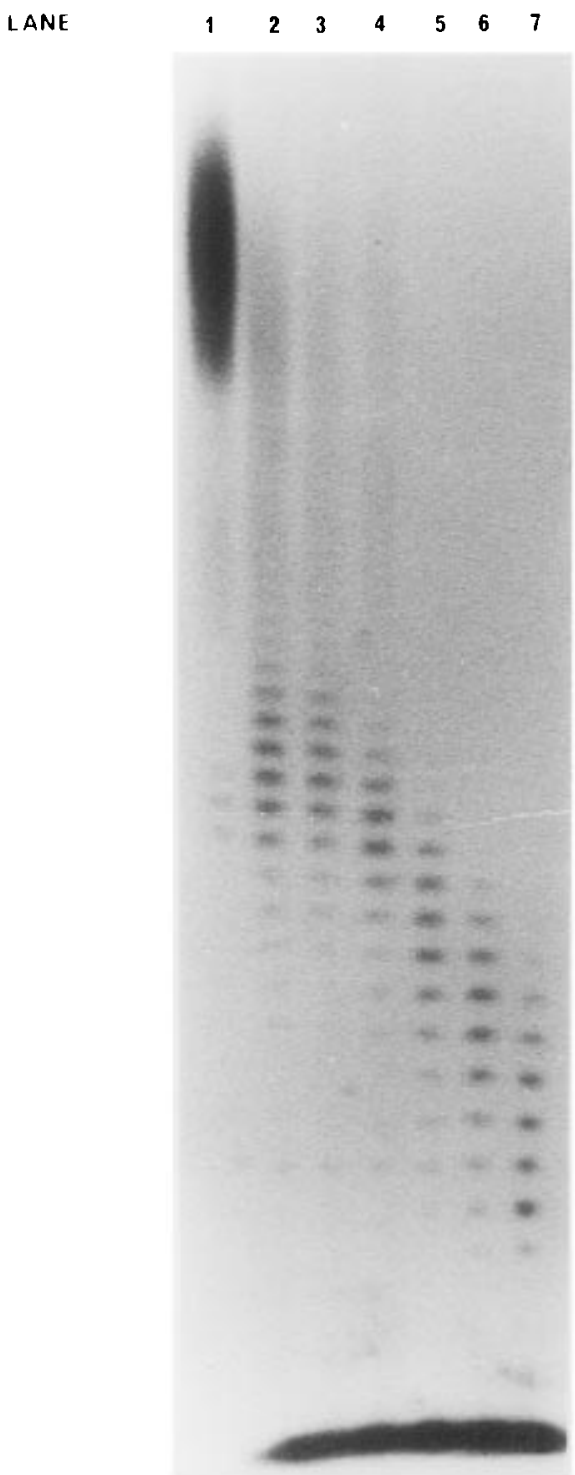

FIGURE 7: Effect of yRPA on the RNase $\mathrm{H}$ activity of $R T H 1$ nuclease. Immunoaffinity purified pol $\alpha$-primase was used to prepare RNA primed poly $(\mathrm{dT})$ templates. The templates were then incubated in the presence of increasing amounts of yRPA (as indicated) and subsequently used as substrates for exonuclease digestion by RTH1. A standard exonuclease assay was carried out using $2.4 \mathrm{ng}$ of $R T H 1$, and the reaction was allowed to proceed for 5 min at $30^{\circ} \mathrm{C}$. Lane 1, blank, no RTH1, no RPA; lane 2, $2.4 \mathrm{ng}$ of $R T H 1$; lane $3, R T H 1$ plus 10 ng of RPA; lane $4, R T H 1$ plus 25 ng of RPA; lane 5, RTH1 plus $50 \mathrm{ng}$ of RPA; lane $6, R T H 1$ plus $100 \mathrm{ng}$ of RPA; lane 7, RTH1 plus $200 \mathrm{ng}$ of RPA.

from the X-ray coordinates is shown in Figure 8A and the computer-generated putative active site model of RTHI nuclease is shown in Figure 8B. The two-dimensional structures of the respective active sites demonstrated a close similarity in the active site structure and also indicated the origin of the RNase $\mathrm{H}$ activity as well as the nuclease activity in general in RTHI and in this family of nucleases. This putative model of the active site would allow design of mutagenesis experiments for further structure function analysis.

\section{DISCUSSION}

The RTH1 nuclease has been demonstrated to be involved in chromosomal DNA replication and perhaps in certain DNA repair processes in eukaryotes (Prakash et al., 1993;
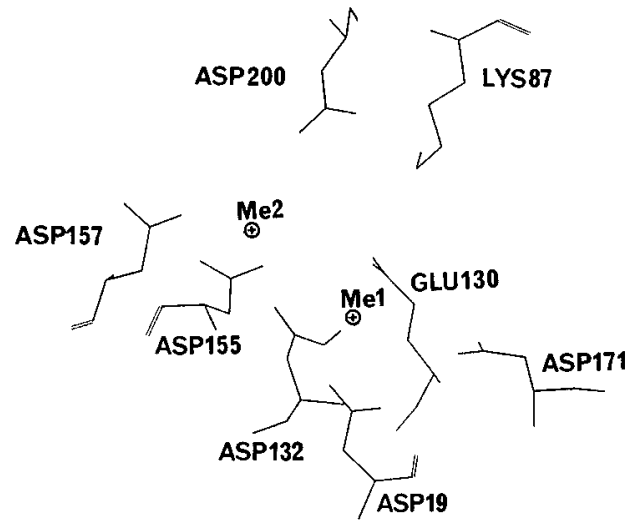

A. T4 RNase $H$
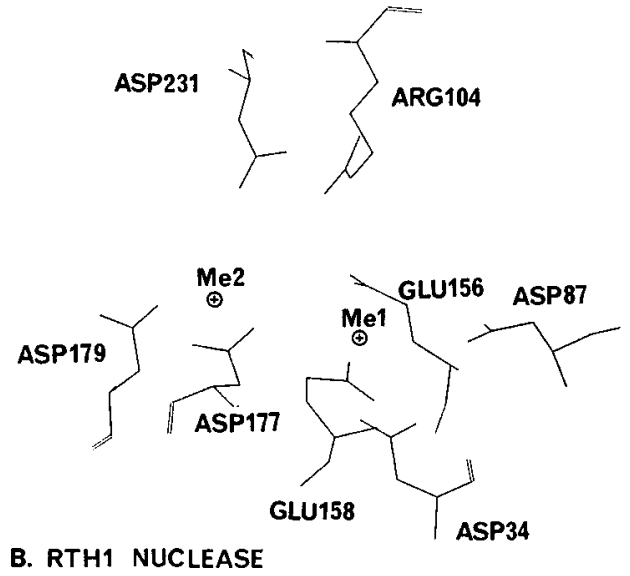

FIGURE 8: Putative model of the active site of RTH1 nuclease. (A) The active site structure of $\mathrm{T} 4 \mathrm{RNase} \mathrm{H}$ as obtained from Meuser et al. (1996). (B) The computer generated model of the active site of $R T H 1$ nuclease. Me1 and $\mathrm{Me} 2$ represent the two metal ions. RTH1 nuclease active site structure was determined by SYBYL program and appropriate force field energy minimization procedure.

Johnson et al., 1995; Sommers et al., 1995). Eukaryotic RPA is also an integral component of the DNA replication apparatus (Wold et al., 1987; Wold \& Kelly, 1988; Erdile et al., 1991). Most DNA replication enzymes act cooperatively with RPA, and in many cases RPA has been shown to stimulate various enzymatic activities. The function of RPA in the endo- and exonuclease function of RTHI nuclease has, thus far, remained unknown. We have explored the possible roles of RPA in the various $R T H I$ nuclease functions, such as flap endonuclease and RNase $\mathrm{H}$ activity.

Inhibitory Effects of RPA on the RTH1 Endonuclease Activity on Short Duplex DNA Substrates. The commonly used endonuclease substrates for RTHI nuclease are the ones first described by Harrington and Lieber (1994) as shown in Figure 1 (structures $\mathrm{c}$ and $\mathrm{d}$ ). Consequently, these substrates were chosen initially to analyze the effects of RPA on the endonuclease activity of RTH1. However, yeast RPA was actually found to inhibit the activity of $R T H 1$ when these substrates were utilized (Figure 2). The duplex region of the substrates as shown in Figure 1 is only $14 \mathrm{bp}$, which is thermodynamically not very stable. Consequently, it is likely that RPA with its limited helix-destabilizing activity could melt or destabilize the DNA duplex and this product would no longer be a substrate for RTH1 nuclease. When this premise was tested experimentally, our results clearly 
demonstrated that RPA is capable of melting the flap and pseudo Y substrates, described earlier by Harrington and Lieber (1994) (Figure 3). The RTH1 nuclease requires a flap structure and is inactive on ssDNA templates, as a result, it would not be able to utilize the substrate once melted by RPA.

RPA Stimulated the Endonuclease Activity on Substrates with Long Duplex Regions. Using M13mp19 ssDNA and synthetic oligonucleotides, we have constructed both flap and pseudo Y substrates with long (50 bp) regions of duplex DNA (structures a and $b$ in Figure 1A). RPA was unable to melt either of these substrates and they were utilized efficiently by the RTHI nuclease (Figure 1B). Thus, these substrates were chosen to test the effect of RPA on the endonuclease activity of RTH1. As shown in Figure 4, RPA stimulated the endonuclease activity on both the flap and pseudo Y substrates. The maximal stimulation was observed with $\geq 50 \mathrm{ng}$ RPA, and the stimulation was approximately 3-fold. In the case of the M13 flap substrate no inhibition was observed with RPA, neither in the range shown or at the higher levels which were examined $(0.2-0.5 \mu \mathrm{g}$ data not shown). However, with the M13 pseudo Y substrate, the endonuclease activity was inhibited at RPA levels $\geq 100$ ng. Since this inhibition was clearly not due to melting of the duplex DNA, we postulated that it was due to the saturation binding of RPA to both strands of the fork, which is possible with a pseudo Y-like structure but not with a flap structure. We have analysed the effect(s) of RPA on the kinetics of the endonuclease reaction. Analysis of the endonuclease activity in the presence and absence of RPA in the 0-30 min time period (Figure 5) suggested that the observed stimulation is a bipartite effect of yRPA on the nuclease reaction: (i) the load time of RTH1 onto the nuclease substrate decreased from 5 to $2 \mathrm{~min}$ in the presence of RPA, and (ii) following initiation of the nuclease reaction, the initial rate of the reaction was 10 -fold higher in the presence of yRPA. Further studies are required to precisely delineate the interaction between yRPA, RTH1, and the DNA substrate and how this leads to such a substantial increase in the initial rate. The yeast RPA appears to be specific for the stimulation of yeast $R T H 1$ nuclease as shown in Figure 6. These results suggested a possible structural interaction between RTH1 nuclease and RPA.

During genomic DNA replication, enzymes and proteins, including RTH1 nuclease, encounter DNA substrates, generally with long duplex regions, such as those present in chromosomal DNA and Okazaki fragments. Consequently, flap structures with small duplex regions are not physiologically significant, and our data indicated that even in that rare event, RPA alone will be able to destroy that structure without any need of RTHI nuclease. Thus, utilization of substrates with long duplex regions would be more worthwhile for mechanistic studies involving RPA or RTHI nuclease.

Effects of RPA on the RNase H Activity of RTH1 nuclease. $R T H 1$ nuclease has a strong $\mathrm{RNase} \mathrm{H}$ activity. It has been shown in $E$. coli that the N-terminal domain $5^{\prime} \rightarrow 3^{\prime}$ exonuclease activity of DNA polymerase I is required for removing primers in vitro and in vivo. Waga and Stillman (1994) demonstrated that the human FEN-1 endonuclease, which was termed maturation factor $1(\mathrm{MF}-1)$ is required for maturation of the Okazaki fragments in the lagging strand.
In the preceding paper, we have shown that $R T H 1$ nuclease can degrade RNA primers synthesized by pol $\alpha$-primase. Consequently, we have examined the effect of RPA on the RNase $\mathrm{H}$ activity of $\mathrm{RTH} 1$ nuclease. A titration of RPA in a RNase $\mathrm{H}$ assay was carried out, and the results are shown in Figure 7. The results of this experiment clearly indicated that the RNase $\mathrm{H}$ activity of $R T H 1$ nuclease is stimulated by RPA.

The three-dimensional structural comparison of RTH1 nuclease with T4 RNase H (Figure 8, panels A and B) indicated a close similarity in the structure and metal ion coordination of the active sites of the two enzymes. This similarity in the structures provided a basis for the RNase $\mathrm{H}$ activity of this multifaceted nuclease.

\section{ACKNOWLEDGMENT}

This work was supported by a grant (GM 36002) from the National Institute of General Medical Sciences, National Institutes of Heath. We thank Dr. Rameshwar Sharma of this University for a critical review of the manuscript and Mr. Kim Sokoloff of the Media Services of this University for excellent photography.

\section{REFERENCES}

Alani, E., Thresher, R., Griffith, J. D., \& Kolodner, R. D. (1992) J. Mol. Biol. 227, 54-71.

Biswas, E. E., Chen, P., Gray, W., Li, Y., Ray, S., \& Biswas, S. B. (1993a) Biochemistry 32, 3013-3019.

Biswas, E. E., Ewing, C., \& Biswas, S. B. (1993b) Biochemistry 32, 3020-3026.

Bradford, M. M. (1976) Anal. Biochem. 72, 248-254.

Charette, M. F., Weaver, D. T., \& DePamphilis, M. L. (1987) Nucleic Acids Res. 14, 3343-3362.

Erdile, L. F., Heyer, W.-D., Kolodner, R. D., \& Kelly T. J. (1991) J. Biol. Chem. 266, 12090-12098.

Goulian, M., Richards, S. H., Heard, C. J., \& Bigsby, B. M. (1990) J. Biol. Chem. 265, 18461-18471.

Gutman, P. D., \& Minton, K. W. (1993) Nucleic Acids Res. 21, 4406-4407.

Guzder, S. N., Habraken, Y., Sung, P., Prakash, L., \& Prakash, S. (1995) J. Biol. Chem. 270, 12973-12976.

Habraken, Y., Sung, P. Prakash, L., \& Prakash, S. (1993) Nature 366, 365-368.

Habraken, Y., Sung, P. Prakash, L., \& Prakash, S. (1994) J. Biol. Chem. 269, 31342-31345.

Habraken, Y., Sung, P. Prakash, L., \& Prakash, S. (1995) J. Biol. Chem. 270, 30194-30198.

Harrington, J. J., \& Lieber, M. R. (1994) Genes Dev. 8, 13441355.

Heyer, W. D., \& Kolodner, R. D. (1989) Biochemistry 28, 28562862.

Heyer, W. D., Rao, M., Erdile, L. F., Kelly, T. J., \& Kolodner, R. D. (1990) EMBO J. 9, 2321-2329.

Hiraoka, L. R., Harrington, J. J., Gerhard, D. S., Lieber, M. R., \& Hsih, C.-L. (1995) Genomics 25, 220-225.

Hollingsworth, H. C., \& Noassal, N. G. (1991) J. Biol. Chem. 266, $1888-1897$.

Ishimi, Y., Claude, A., Bullock, P., \& Hurwitz, J. (1988) J. Biol. Chem. 263, 19723-19733.

Johnson, R. E., Gopala, K. K., Prakash, L., \& Prakash, S. (1995) Science 269, 238-240.

Kohlstaedt, L. A., Wang, J., Friedman, J. M., Rice, P. A., \& Steitz, T. A. (1992) Science 256, 1783-1790.

Kornberg, A., \& Baker, T. A. (1992) DNA Replication, Freeman, San Francisco, CA.

Lebowitz, J. H., \& McMacken, R. M. (1986) J. Biol. Chem. 261, 4738-4748. 
Mueser, T. C., Nossal, N. B., \& Hyde, C. C. (1996) Cell 85, $1101-$ 1112.

Murray, J. M., Tavassoli, M., Al-Harithy, R., Sheldrick, K. S., Lehman, A. R., Carr, A. M., \& Watts, F. Z. (1994) Mol. Cell. Biol. 4878-4888.

O'Donovan, A., Davies, A. A., Moggs, J. G., West, S. C., \& Wood, R. D. (1994) Nature 371, 432-435.

Plevani, P., Foiani, M., Valsasnini, P., Badaracco, G., Cheriathundam, E., \& Chang, L. M. S. (1985) J. Biol. Chem. 260, 81738181.

Prakash, L., Prakash, S., \& Sung, P. (1993) Annu. Rev. Genet. 27, 33-70.

Robins, P., Pappin, D. J. C., Wood, R. D., \& Lindahl, T. (1994) J. Biol. Chem. 269, 28535-28538.

Scherly, D., Nouspikel, T., Corlet, J., Ucia, C., Bairoch, A., \& Clarkson, S. G. (1993) Nature 363, 182-185.
Shen, B., Nolan, J. P., Sklar, L. A., \& Park, M. S. (1996) J. Biol. Chem. 16, 9173-9176.

Sommers, C. H., Miller, E. J., Dujon, B., Prakash, S., \& Prakash, L. (270) J. Biol. Chem. 270, 4193-4196.

Turchi, J. J., Huang, L., Murante, R. S., Kim, Y., \& Bambara, R. A. (1994) Proc. Natl. Acad. Sci. U.S.A. 91, 9803-9807.

Waga, S., Bauer, G., \& Stillman, B. (1994) J. Biol. Chem. 269, 10923-10934.

Wold, M. S., \& Kelly, T. J. (1988) Proc. Natl. Acad. Sci. U.S.A. 85, 2523-2527.

Wold, M. S., Li, J. J., \& Kelly, T. J. (1987) Proc. Natl. Acad. Sci. U.S.A. 84, 3643-3647.

Zhu, F. X., Biswas, E. E., \& Biswas, S. B. (1997) Biochemistry 36, 5947-5954.

BI962890U 